\title{
THE TOPOLOGICAL COMPLEMENTATION THEOREM ì LA ZORN
}

\author{
PAUL S. SCHNARE
}

\begin{abstract}
Steiner's topological complementation theorem is given a short simple proof using Zorn's Lemma.
\end{abstract}

A. K. Steiner [3, Theorem 7.8, p. 397] proved that the lattice of topologies on a fixed set $X$, denoted $\Sigma$ or $\Sigma(X)$, is complemented. ${ }^{1}$ In fact, she showed that each $t \in \Sigma$ has a complement in $I=I I(X)$, the sublattice of principal topologies. (A topology $t \in \Sigma$ is principal iff each point $x \in X$ has a smallest $t$-neighborhood: $B_{t}(x)$.) Her proof was quite complicated and although van Rooij [1] gave a simpler proof, his proof used both Zorn's Lemma and two applications of transfinite induction. The purpose of this note is to prove Steiner's Theorem via a standard Zornification by the simple trick of suitably adjoining a new point $p$ to $X$ and subsequently discarding it.

Theorem (A. K. Steiner). Every $t \in \Sigma$ has a complement $t^{\prime} \in \Pi$.

Proof. Take a point $p \notin X$ and let $T$ be the topology defined on $Y=X \cup\{p\}$ by $T=t \cup\{U \cup\{p\} \mid U \in t\}$. Let $\mathscr{A}=\{(A, s) \mid p \in A \subset Y$ and $s \in \Pi(A)$ is a complement for $T \mid A\}$. Then $\mathscr{A} \neq \varnothing$, since $(\{p\},\{\varnothing,\{p\}\}) \in$ $\mathscr{A}$. Partially order $\mathscr{A}$ by $\left(A_{1}, s_{1}\right) \leqq\left(A_{2}, s_{2}\right)$ iff (i) $A_{1} \subset A_{2}$, (ii) $B_{1}(x)=B_{2}(x)$ for $x \in A_{1} \backslash\{p\}$, and (iii) $B_{1}(p) \subset B_{2}(p) \subset B_{1}(p) \cup A_{2} \backslash A_{1}$. If

$$
\mathscr{B}=\left\{\left(A_{i}, s_{i}\right) \mid i \in I\right\} \subset \mathscr{A}
$$

is totally ordered, let $(A, s)$ be defined by $A=\bigcup A_{i} ; B_{s}(x)=B_{i}(x)$ if $x \in A_{i} \mid\{p\}$ and $B_{s}(p)=\bigcup B_{i}(p)$. It is easily verified that $(A, s)$ is an upper bound for $\mathscr{B}$ in $\mathscr{A}$, so by Zorn's Lemma $\mathscr{A}$ has a maximal element, say $(M, m)$. But, $M=Y$. For otherwise, if $q \in Y \backslash M$ we can extend $m$ to $m^{\prime}$ on

Received by the editors November 8, 1971 .

AMS 1969 subject classifications. Primary 5420, 0635.

Key words and phrases. Topological complementation theorem, lattice of topologies, principal topologies, complemented lattice.

${ }^{1}$ For $t_{1}, t_{2} \in \Sigma$ we have $t_{1} \leqq t_{2}$ iff $t_{1} \subset t_{2}$. We say that $t^{\prime} \in \mathcal{\Sigma}$ is a complement for $t \in \Sigma$ iff $t \vee t^{\prime}=1$, the discrete topology, and $t \wedge t^{\prime}=0$, the trivial topology. See [2] for the cardinality of the set of complements.

(c) American Mathematical Society 1972 
$M^{\prime}=M \cup\{q\}$ by: $B_{m^{\prime}}(p)=B_{m}(p)$ if $M$ is not open in $M^{\prime}, B_{m^{\prime}}(p)=B_{m}(p) \cup$ $\{q\}$ if $M$ is open in $M^{\prime}, B_{m^{\prime}}(q)=\{q\}$ if $\{q\}$ is not open in $M^{\prime}, B_{m^{\prime}}(q)=$ $B_{m}(p) \cup\{q\}$ if $\{q\}$ is open in $M^{\prime}$. Since $(M, m) \leqq\left(M^{\prime}, m^{\prime}\right) \in \mathscr{A}$, this is a contradiction. It immediately follows that, since $m$ is a principal complement for $T$ and since both $X$ and $\{p\}$ are $T$-open, $m \mid X$ is a principal complement for $t$.

\section{REFERENCES}

1. A. C. M. van Rooij, The lattice of all topologies is complemented, Canad. J. Math. 20 (1968), 805-807. MR 37 \#3504.

2. P. S. Schnare, Infinite complementation in the lattice of topologies, Fund. Math. 64 (1969), 249-255. MR 39 \#3444.

3. A. K. Steiner, The lattice of topologies: Structure and complementation, Trans. Amer. Math. Soc. 122 (1966), 379-398. MR 32 \#8303.

Department of Mathematics, University of Florida, Gainesville, Florida 